\title{
Adjusting Overall Survival Estimates for Treatment Switching in Metastatic, Castration-Sensitive Prostate Cancer: Results from the LATITUDE Study
}

\author{
Susan Feyerabend ${ }^{1} \cdot$ Fred Saad $^{2} \cdot$ Nolen Joy Perualila $^{3}$ (i) . Suzy Van Sanden ${ }^{3} \cdot$ Joris Diels $^{3} \cdot$ Tetsuro Ito $^{4}$. \\ Peter De Porre ${ }^{5} \cdot$ Karim Fizazi ${ }^{6}$
}

Published online: 21 November 2019

(c) The Author(s) 2019

\begin{abstract}
Background LATITUDE was the first phase 3 trial examining the survival benefit of adding abiraterone acetate (AA) + prednisone $(\mathrm{P})$ to androgen-deprivation therapy (ADT) in newly diagnosed metastatic, castration-sensitive prostate cancer (mCSPC). Due to significant improvement in overall survival after the first interim analysis, patients in the placebos + ADT arm could switch to AA $+\mathrm{P}+\mathrm{ADT}$ during an open-label extension. As in other studies where switching is allowed, statistical adjustments are needed to assess the real benefit of new drugs.

Patients and Methods This was a post hoc analysis to estimate the true survival benefit of AA $+\mathrm{P}+\mathrm{ADT}$ in patients with newly diagnosed mCSPC by applying statistical adjustments commonly used to adjust for treatment switching.

Results Of 112 patients still receiving placebos + ADT at the first interim analysis, 72 switched to AA + P + ADT during the open-label extension. Final analysis was conducted after median follow-up of 51.8 months. Compared to the placebos $+\mathrm{ADT}$ arm, the risk of death in the AA $+\mathrm{P}+\mathrm{ADT}$ arm was $34 \%$ lower [hazard ratio $(\mathrm{HR})=0.663$ (95\% confidence interval 0.566-0.778)] by unadjusted intent-to-treat analysis, $37 \%$ lower [HR $=0.629$ (95\% confidence interval $0.526-0.753)$ ] by rank preserving structure failure time modeling, and $38 \%$ lower $[\mathrm{HR}=0.616$ (95\% confidence interval $0.524-0.724)]$ by inverse probability of censoring weights.

Conclusions Analyses adjusting for treatment switching using two different statistical approaches confirm the improved survival benefit of adding AA + P to ADT in patients with newly diagnosed mCSPC.
\end{abstract}

Trial Registration ClinicalTrials.gov identifier NCT01715285.

Electronic supplementary material The online version of this article (https://doi.org/10.1007/s11523-019-00685-x) contains supplementary material, which is available to authorized users.

Nolen Joy Perualila

NPeruali@its.jnj.com

1 Urologic Oncology, Studienpraxis Urologie, Nürtingen, Germany

2 Urologic Oncology, Centre Hospitalier de L'Université de Montréal/CRCHUM, Montréal, QC, Canada

3 Health Economics and Market Access EMEA, Janssen, Beerse, Belgium

4 Health Economics and Market Access EMEA, Janssen, High Wycombe, Buckinghamshire, UK

5 Clinical Oncology, Janssen Research \& Development BE, Spring House, USA

6 Gustave Roussy, University of Paris Sud, Paris, France

\section{Key Points}

The LATITUDE trial showed that adding abiraterone acetate + prednisone to androgen-deprivation therapy (ADT) provided a significant survival benefit in men with newly diagnosed metastatic, castration-sensitive prostate cancer.

Due to significant improvement in overall survival after the first interim analysis, patients receiving placebos + ADT arm could switch to abiraterone acetate + prednisone + ADT during an open-label extension.

This post hoc analysis confirmed the significant benefit of adding abiraterone acetate + prednisone to ADT following adjustment for bias introduced by treatment switching. 


\section{Introduction}

Prostate cancer is the second-most common cancer worldwide in men, accounting for $15 \%$ of all cancers diagnosed in 2012, and it is the fifth-leading cause of death [1]. The incidence of prostate cancer is highest in developed countries, where it is the most common cancer in men. This high incidence is thought to be mostly due to regular screening for prostate-specific antigen and subsequent biopsy. About $15-25 \%$ of patients newly diagnosed with prostate cancer have metastatic disease [2-4], which has a poor prognosis and a 5-year survival rate below 30\% [5].

For nearly 80 years, the standard of care for patients with newly diagnosed metastatic prostate cancer has been androgen-deprivation therapy (ADT), which consists of a luteinizing hormone-releasing hormone agonist (medical castration) or orchiectomy (surgical castration) with or without concurrent anti-androgens [6]. However, patients eventually become castration-resistant and need additional drugs to control the cancer. Clinical trials in the early 2000s indicated that docetaxel was effective for treating metastatic castration-resistant prostate cancer, and following trials showed that docetaxel is even more effective for metastatic, castration-sensitive prostate cancer (mCSPC) [6]. Based on this proven survival benefit, the addition of docetaxel to ADT has since become a standard of care for patients with mCSPC [7]. Docetaxel, however, causes frequent grade 3-5 toxicity, including neutropenia, febrile neutropenia, and fatigue, limiting its use, especially in patients with advanced age, poor performance status, or coexisting illnesses [6]. Also, studies have not shown a conclusive survival benefit of adding docetaxel to ADT for patients with low-volume disease [7].

More recently, abiraterone acetate (AA) plus prednisone (P) in combination with ADT has been added as a standard of care for mCSPC [7] based on a proven overall survival (OS) benefit $[8,9]$. AA is a prodrug of abiraterone, a selective inhibitor of testosterone biosynthesis that acts by blocking cytochrome P450 c17 [6]. Network meta-analyses suggest that $\mathrm{AA}+\mathrm{P}+\mathrm{ADT}$ improves survival at least as well as docetaxel + ADT and is better at preventing disease progression and improving quality of life [10,11]. Current consensus recommendations are that $\mathrm{AA}+\mathrm{P}$ in combination with ADT should be considered for patients with newly diagnosed mCSPC who are fit enough for the regimen [12, 13]. For patients with $\mathrm{mCSPC}$, the recommended dose of AA is $1,000 \mathrm{mg}$ orally once daily with $5 \mathrm{mg}$ prednisone orally once daily [14].

LATITUDE was the first phase 3 trial examining the survival benefit of adding $\mathrm{AA}+\mathrm{P}$ to ADT. It was a multinational, double-blind, randomized, placebo-controlled study in 1,199 men with newly diagnosed mCSPC [8]. The study was unblinded shortly after the first interim analysis on 31
October 2016 after a median follow-up of 30.4 months due to significant and clinically meaningful improvement in OS [hazard ratio $(\mathrm{HR})=0.62(95 \%$ confidence interval $(\mathrm{CI})$ $0.51-0.76)]$ and radiographic progression or death $[\mathrm{HR}=0.47$ (95\% CI 0.39-0.55)]. Patients in the AA + P + ADT arm also had less pain and fatigue and better overall health-related quality of life than patients in the placebos + ADT arm [15]. As a result, patients in the placebos + ADT arm of LATITUDE were allowed to switch to $\mathrm{AA}+\mathrm{P}+\mathrm{ADT}$ during an open-label extension.

In the final analysis, after a median follow-up of 51.8 months, OS was longer in the $\mathrm{AA}+\mathrm{P}+\mathrm{ADT}$ arm than in the placebos + ADT arm [median, 53.3 months $(95 \% \mathrm{CI}$ 48.2-not reached) vs. 36.5 months (95\% CI 33.5-40.0); $\mathrm{HR}=0.66(95 \% \mathrm{CI} 0.56-0.78)]$ [16]. Because the placebo arm may have gained survival time attributed to $\mathrm{AA}+\mathrm{P}$ during the open-label extension, the clinical benefit associated with AA + P may have been underestimated. Adjusting OS benefits for treatment switching is common in oncology [17], and a variety of methods are available [18, 19]. Here, we conducted a post hoc analysis to estimate the true OS benefit of $\mathrm{AA}+\mathrm{P}+\mathrm{ADT}$ by applying statistical adjustments to the final results.

\section{Methods}

\subsection{Study Design}

This was a post hoc analysis examining OS following adjustment for treatment switching in patients enrolled in the LATITUDE study (NCT01715285) [8]. The LATITUDE study was conducted at 235 sites in 34 countries in Europe, the Asia-Pacific region, Latin America, and Canada. Patients $\geq 18$ years of age with high-risk, newly diagnosed mCSPC ( $\leq 3$ months before randomization) and an Eastern Cooperative Oncology Group performance status score of 0-2 were included. Diagnosis of $\mathrm{mCSPC}$ had to be demonstrated by a positive bone scan or metastatic lesions at the time of diagnosis on computed tomography or magnetic resonance imaging according to RECIST, version 1.1. Patients were considered at high risk if they had at least two of the following factors associated with poor prognosis: Gleason score $\geq 8, \geq 3$ bone lesions, and presence of measurable visceral metastasis. Patients were randomized in a 1:1 ratio to receive AA (1000 mg once daily as four 250-mg tablets) and P (5 mg once daily) or placebos in addition to ADT. The trial was conducted according to the principles of the Declaration of Helsinki and the International Council for Harmonisation Good Clinical Practice guidelines. All patients provided written informed consent before participating in the trial.

An initial interim analysis was performed after observing $50 \%$ of the total number of required death events for the final 


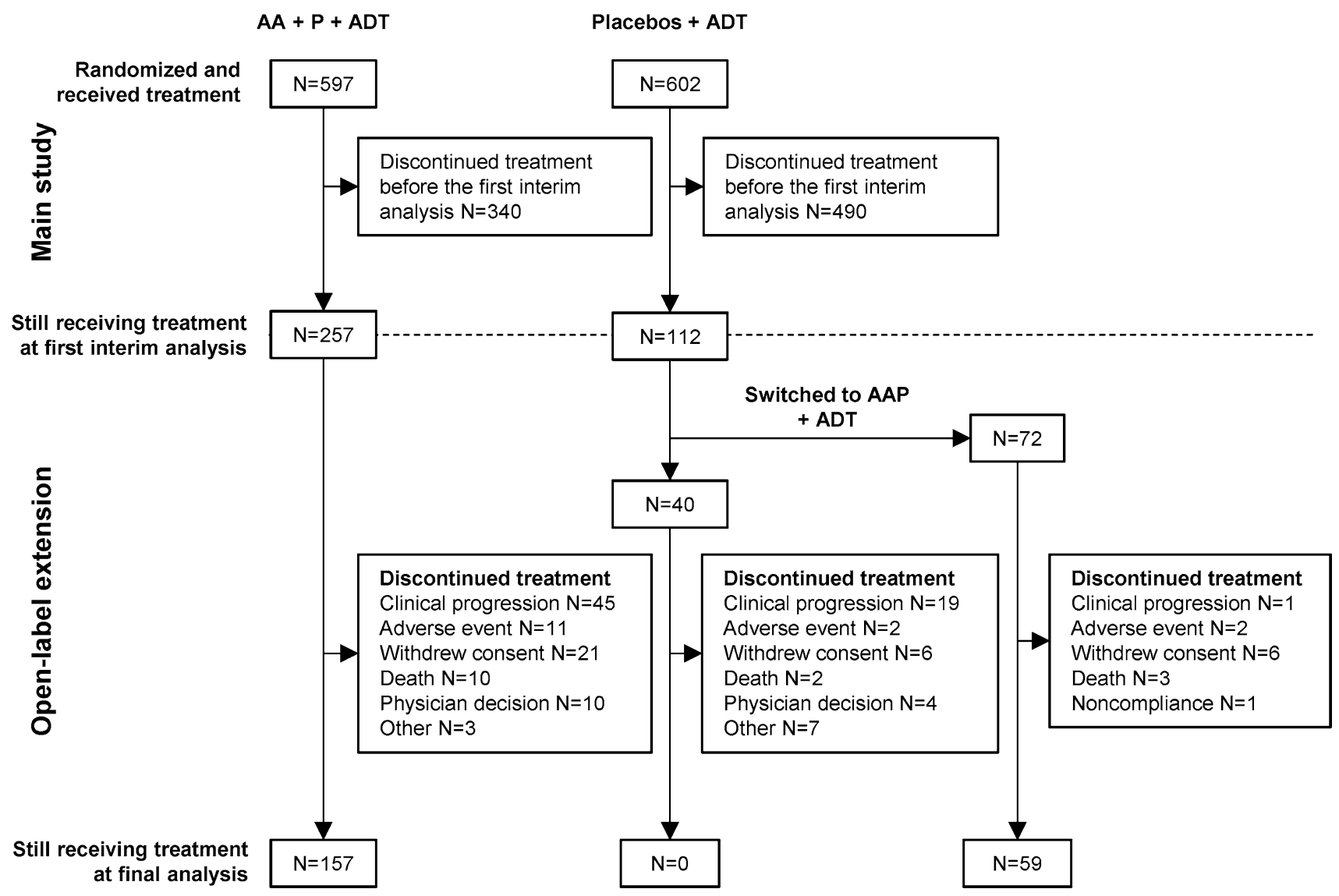

Fig. 1 Disposition. Patients were randomized in a 1:1 ratio to receive $\mathrm{AA}+\mathrm{P}+\mathrm{ADT}$ or placebos + ADT. Because of a positive result at the first interim analysis, patients had the opportunity to enroll in an open-label extension phase in which they received AA $+\mathrm{P}+\mathrm{ADT}$ for up to 3 years. Patients who had previously received placebos could

analysis. Because of a positive result at the first interim analysis, as determined by an independent data and safety monitoring committee, patients remaining in the placebos arm at the time of unblinding were eligible to switch to the active drug $(\mathrm{AA}+\mathrm{P})$ in an open-label extension phase for up to 3 years.

\subsection{Statistical Analysis}

Statistical analyses were performed using SAS version 9.4 (SAS Institute, Cary, NC, USA) in the intent-to-treat population, which included all randomized patients. The endpoint in this post hoc analysis was OS, a co-primary endpoint in the LATITUDE trial. OS was defined as the time from randomization to death from any cause. Survival distribution and median OS were estimated by Kaplan-Meier analysis. HRs and associated $95 \%$ CIs were estimated using an unstratified Cox proportional hazards model. Rank preserving structure failure time modeling (RPSFTM) [20] and inverse probability of censoring weights (IPCW) [21] were used to adjust survival estimates for treatment switching from the placebos switch to the $\mathrm{AA}+\mathrm{P}$ arm if they met the entry eligibility requirements for hepatic function and cardiovascular disease before starting $\mathrm{AA}+\mathrm{P}$. "Deaths" refers to discontinuation of treatment due to death only. $A A$ abiraterone acetate, $A D T$ androgen-deprivation therapy, $P$ prednisone

to $\mathrm{AA}+\mathrm{P}$. The cut-off for statistical significance was a $P$ value $<0.05$. Details of RPSFTM and IPCW development and conduct are provided in the Supplemental Methods.

\section{Results}

The LATITUDE study included 1,199 patients, of whom 597 were randomized to AA + P + ADT and 602 to placebos + ADT [8]. The first interim analysis of OS was performed when 406 deaths had occurred (31 October 2016) with a median follow-up time of 30.4 months [8]. The final analysis of OS was performed on 15 August 2018, with a median follow-up of 51.8 months [16].

Baseline characteristics, patient disposition, and reasons for discontinuation for the LATITUDE trial were previously published $[8,16]$. Briefly, at the first interim analysis, treatment was ongoing for 257 of $597(43.0 \%)$ patients in the $\mathrm{AA}+\mathrm{P}+\mathrm{ADT}$ arm and 112 of 602 (18.6\%) patients in the placebos + ADT arm (Fig. 1). Of the 112 patients still receiving 
Fig. 2 Treatment timeline of patients who switched from placebos + ADT to AA + P+ADT. Clinical progression was defined as deterioration of ECOG performance status to grade 3 or higher or need to initiate any of the following because of tumor progression (even in the absence of radiographic evidence of disease): anticancer therapy for prostate cancer; radiation therapy or surgical interventions for complications due to tumor progression; or cancer pain requiring immediate administration of chronic opioid analgesics. If a patient had radiographic progression (without clinical progression) and alternate therapy was not initiated, treatment could continue at the discretion of the investigator. "Death" refers to discontinuation of treatment due to death only. Radiographic progression was defined as detection of soft tissue lesions by computed tomography/ magnetic resonance imaging per RECIST 1.1 or by bone lesion progression on bone scans per modified Prostate Cancer Working Group 2 criteria. $A A$ abiraterone acetate, $A D T$ androgen deprivation therapy, $E C O G$ Eastern Cooperative Oncology Group, $P$ prednisone

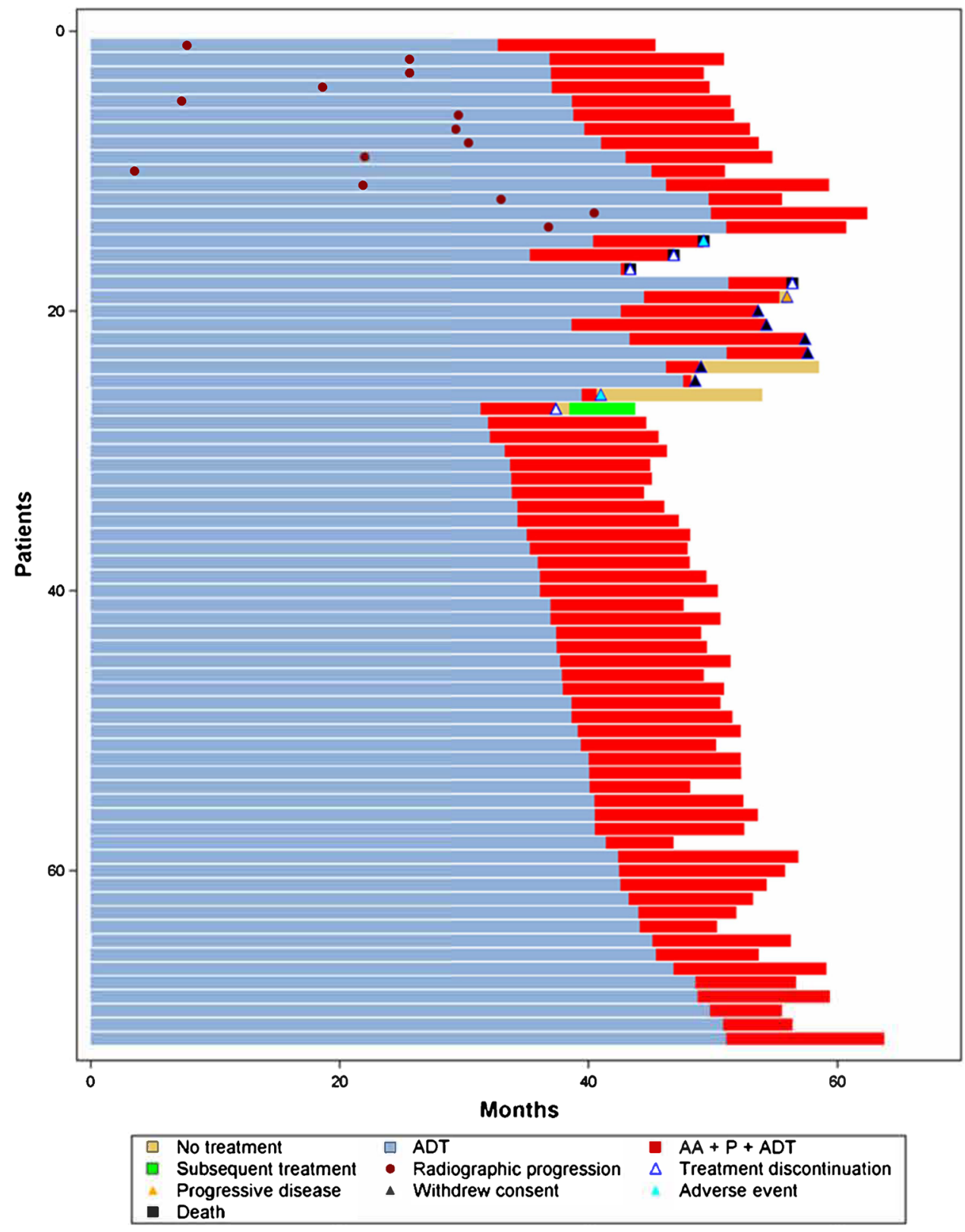

placebos $+\mathrm{ADT}, 72$ switched over to the $\mathrm{AA}+\mathrm{P}+\mathrm{ADT}$ during the open-label extension after a median of 40.07 months (interquartile range 36.98-44.34). Between the first interim analysis and the final analysis, 13 of the 72 patients who switched from placebos + ADT discontinued AA + P + ADT, and the remaining 59 were still receiving $\mathrm{AA}+\mathrm{P}+\mathrm{ADT}$ at the time of the final analysis (Fig. 2). The median for time from switching to death or end of the trial was 11.78 months (interquartile range 9.85-13.71). The 40 patients who did not switch to AA + P + ADT remained on placebos + ADT. Of these 40 , none were still receiving treatment at the end of the study. In all groups, the main reason for discontinuation was clinical progression.
Patients who switched from the placebos + ADT arm to the $\mathrm{AA}+\mathrm{P}+\mathrm{ADT}$ arm resided in all regions and were in all age groups (Table 1). Eastern Cooperative Oncology Group performance status, total Gleason scores, serum prostate-specific antigen, lactate dehydrogenase, and presence of visceral disease were similar between switchers, non-switchers, and patients originally randomized to the $\mathrm{AA}+\mathrm{P}+\mathrm{ADT}$ arm. Pain scores and numbers of bone lesions, however, were lower in switchers than non-switchers and patients in the $\mathrm{AA}+\mathrm{P}+\mathrm{ADT}$ arm.

In the final unadjusted intent-to-treat analysis, the median OS was 53.3 months (95\% CI 48.2-not reached) in the $\mathrm{AA}+\mathrm{P}+\mathrm{ADT}$ arm and 36.5 months (95\% CI 33.5-40.0) in the placebos +ADT arm (Fig. 3). This corresponded to a $34 \%$ 
Table 1 Patient characteristics

\begin{tabular}{|c|c|c|c|}
\hline \multirow[t]{3}{*}{ Characteristics } & \multirow{3}{*}{$\begin{array}{l}\mathrm{AA}+\mathrm{P}+\mathrm{ADT} \\
\text { Did not switch } \\
N=597\end{array}$} & \multicolumn{2}{|l|}{ Placebos + ADT } \\
\hline & & \multirow{2}{*}{$\begin{array}{l}\text { Did not switch } \\
N=530\end{array}$} & \multirow{2}{*}{$\begin{array}{l}\text { Switched to } \\
\text { AA + P + ADT } \\
N=72\end{array}$} \\
\hline & & & \\
\hline Age (years), mean (SD) & $67.3(8.48)$ & $66.8(8.69)$ & $67.2(8.96)$ \\
\hline \multicolumn{4}{|l|}{ Age category (years), $n(\%)$} \\
\hline$<65$ & $221(37.0)$ & 209 (39.4) & $24(33.3)$ \\
\hline $65-69$ & $112(18.8)$ & $113(21.3)$ & $21(29.2)$ \\
\hline $70-74$ & $141(23.6)$ & $105(19.8)$ & $10(13.9)$ \\
\hline$\geq 75$ & $123(20.6)$ & $103(19.4)$ & $17(23.6)$ \\
\hline \multicolumn{4}{|l|}{ ECOG performance status at baseline, $n(\%)$} \\
\hline 0 or 1 & $573(96.0)$ & $515(97.2)$ & $71(98.6)$ \\
\hline 2 & $24(4.0)$ & $15(2.8)$ & $1(1.4)$ \\
\hline \multicolumn{4}{|l|}{ Region, $n(\%)$} \\
\hline Asia & $124(20.8)$ & $103(19.4)$ & $18(25.0)$ \\
\hline Eastern Europe & $214(35.8)$ & $192(36.2)$ & $25(34.7)$ \\
\hline Rest of world & $104(17.4)$ & $84(15.8)$ & $18(25.0)$ \\
\hline Western Europe & $155(26.0)$ & $151(28.5)$ & $11(15.3)$ \\
\hline Total Gleason score, mean (SD) & $8.6(0.68)$ & $8.6(0.67)$ & $8.4(0.6)$ \\
\hline \multicolumn{4}{|l|}{ Gleason score category, $n(\%)$} \\
\hline$<8$ & $13(2.2)$ & $15(2.8)$ & $1(1.4)$ \\
\hline$\geq 8$ & $584(97.8)$ & $515(97.2)$ & $71(98.6)$ \\
\hline \multicolumn{4}{|l|}{ Presence of visceral disease, $n(\%)$} \\
\hline Yes & $114(19.1)$ & $103(19.4)$ & $11(15.3)$ \\
\hline No & $483(80.9)$ & $427(80.6)$ & $61(84.7)$ \\
\hline \multicolumn{4}{|l|}{ Baseline pain score category, $n(\%)^{\mathrm{a}}$} \\
\hline $0-1$ & $284(49.7)$ & $247(48.1)$ & $41(60.3)$ \\
\hline $2-3$ & $125(21.9)$ & $120(23.4)$ & $18(26.5)$ \\
\hline$\geq 4$ & $163(28.5)$ & $146(28.5)$ & $9(13.2)$ \\
\hline \multicolumn{4}{|l|}{ Bone lesions at baseline, $n(\%)$} \\
\hline$\leq 10$ & $211(35.3)$ & $180(34.0)$ & $41(56.9)$ \\
\hline$>10$ & $386(64.7)$ & $350(66.0)$ & $31(43.1)$ \\
\hline Log baseline serum PSA (ng/mL), mean (SD) & $3.3(2.33)$ & $3.2(2.18)$ & $3.1(2.33)$ \\
\hline Log baseline lactate dehydrogenase (U/L), mean (SD) & $5.2(0.3)$ & $5.2(0.3)$ & $5.1(0.17)$ \\
\hline Log baseline hemoglobin (g/L), mean (SD) & $4.9(0.14)$ & $4.9(0.14)$ & $4.9(0.11)$ \\
\hline
\end{tabular}

For some characteristics, the total number of patients was less than $N$ due to missing values

$A A$ abiraterone acetate, $A D T$ androgen-deprivation therapy, ECOG Eastern Cooperative Oncology Group, $P$ prednisone, $P S A$ prostate-specific antigen, $S D$ standard deviation

${ }^{\text {a }}$ Two patients in each trial arm with missing values at the first interim analysis have updated values at the second interim analysis

lower risk of death in the $\mathrm{AA}+\mathrm{P}+\mathrm{ADT}$ arm than in the placebos + ADT arm $[\mathrm{HR}=0.663(95 \%$ CI $0.566-0.778)]$. When adjusted by RPSFTM, the median OS in the placebos + ADT arm was 35.1 months (95\% CI 32.6-38.8), corresponding to a $38 \%$ lower risk of death in the $\mathrm{AA}+\mathrm{P}+\mathrm{ADT}$ arm than in the placebos + ADT arm (HR $=0.616$ (95\% CI, 0.524-0.724)). When adjusted by IPCW, the median OS was 36.0 months (95\% CI 33.3-39.6) in the placebos + ADT arm, corresponding to a $37 \%$ lower risk of death in the AA+P+ADT arm than in the placebos + ADT $\operatorname{arm}[\mathrm{HR}=0.629(95 \%$ CI $0.526-0.753)]$.

\section{Discussion}

The final analysis of LATITUDE indicated a significant OS benefit from adding AA $+\mathrm{P}$ to ADT in men with newly diagnosed mCSPC [16]. The current post hoc analysis confirmed the significant benefit of adding AA $+\mathrm{P}$ to ADT after adjustment for bias introduced by switching from placebos to $\mathrm{AA}+\mathrm{P}$ during the open-label extension. 


\section{a}

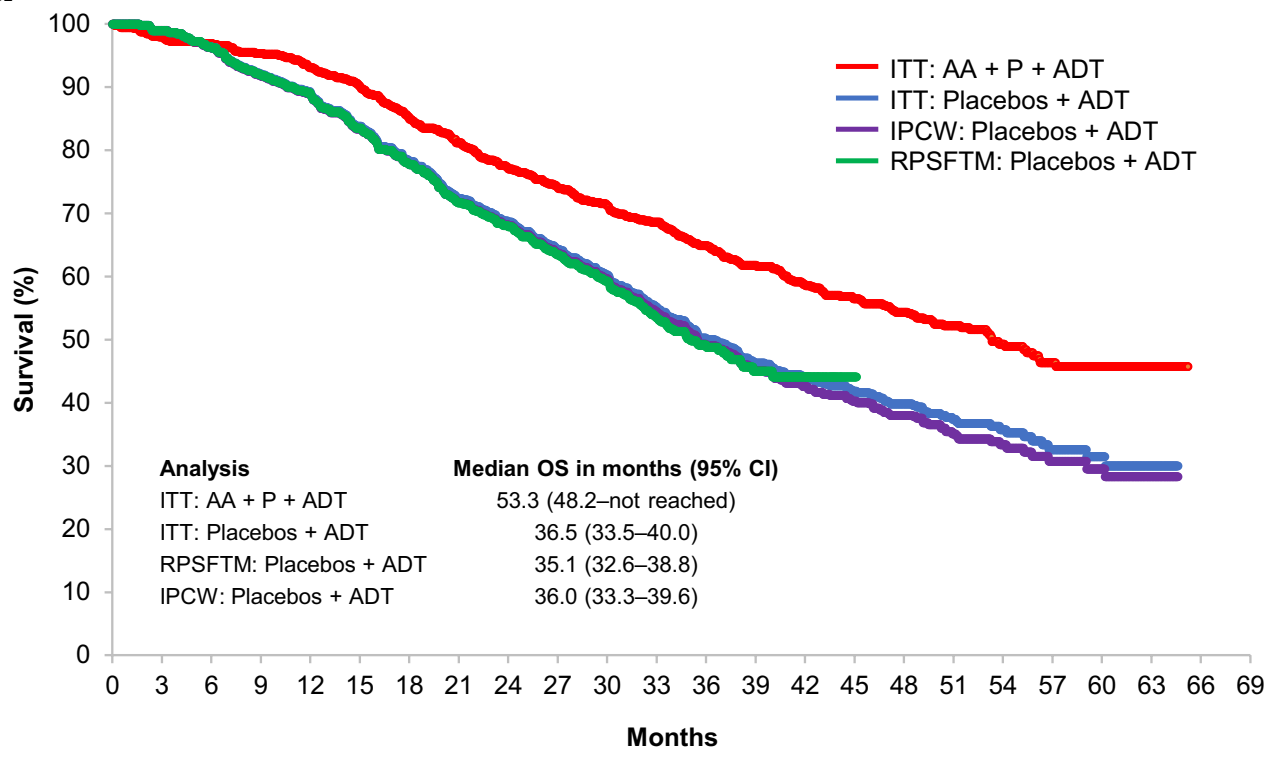

No. of patients still at risk

ITT: AA + P + ADT $597580565551529509479450425406389373 \quad 351332311286240 \quad 185124 \quad 77 \quad 40 \quad 8 \quad 0$

ITT: Placebos + ADT $602 \begin{array}{lllllllllllllllllllllll}590 & 564 & 527 & 505 & 469 & 432 & 392 & 368 & 338 & 315 & 287 & 257 & 232 & 220 & 197 & 165 & 117 & 69 & 43 & 23 & 4 & 0\end{array}$

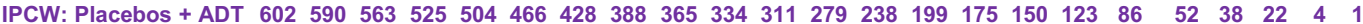
RPSFTM: Placebos + ADT $602585552519492453416 \quad 379352 \quad 325299227 \quad 132 \quad 65 \quad 26 \quad 1 \quad 0$

b

$\begin{array}{lcc} & \text { HR }(95 \% \text { Cl }) & \text { P-value } \\ \begin{array}{l}\text { ITT first interim } \\ \text { analysis }\end{array} & 0.625(0.513-0.762) & <0.0001 \\ \text { ITT final analysis } & 0.663(0.566-0.778) & <0.0001 \\ \text { IPCW } & 0.616(0.524-0.724) & <0.0001 \\ \text { RPSFTM } & 0.629(0.526-0.753) & <0.0001\end{array}$

Fig. 3 Kaplan-Meier curves for overall survival. a Survival distribution and median overall survival were estimated by Kaplan-Meier analysis. b HRs and associated 95\% CIs were estimated using an unstratified Cox proportional hazards model. $A A$ abiraterone acetate,

Adjusting for treatment switching is necessary to assess the real benefit of new drugs, especially in oncology [17]. For example, when patients switch from placebo to an active therapy, as in this study, the true OS benefit may be underestimated. Several methods can be used to adjust for bias due to treatment switching $[18,19]$. In the current analysis, we employed RPSFTM and IPCW, which have been used to adjust for treatment switching in clinical trials of antineoplastic agents [22-25]. IPCW works by censoring patients when they switch from placebo to experimental treatment
$A D T$ androgen-deprivation therapy, $C I$ confidence interval, $H R$ hazard ratio, IPCW inverse probability of censoring weights, ITT intent to treat, $O S$ overall survival, $P$ prednisone, RPSFTM rank preserving structure failure time model

and then, to adjust for any differences in characteristics from the original study population, up-weighting any patients who did not switch and who had similar characteristics as the patients who switched [21]. For this to be valid, all prognostic covariates must be taken into account, which is referred to as the "no unmeasured confounders" assumption. Although we took a wide range of confounders into account in the current analysis, it is not possible to be certain that there were no unmeasured confounders. RPSFTM, in contrast, attempts to estimate the "counterfactual" survival 
times that would have been observed had treatment switching not occurred [22]. The main assumption for RPSFTM is that there is a "common treatment effect" [20]. For this study, that means that the benefit of $\mathrm{AA}+\mathrm{P}$ was assumed to be similar for patients in the placebo arm who later switched to $\mathrm{AA}+\mathrm{P}$ as for patients who received $\mathrm{AA}+\mathrm{P}$ starting from baseline. The common treatment effect may be a reasonable approximation here because adding $\mathrm{AA}+\mathrm{P}$ to ADT should have had a similar effect in patients randomized to $\mathrm{AA}+\mathrm{P}+\mathrm{ADT}$ at the beginning of the trial as in patients whose disease had not progressed while receiving placebos + ADT and who switched to AA + P+ADT. Although it is possible that patients who switched from placebo benefitted more from $\mathrm{AA}+\mathrm{P}$ because they had a better response to ADT alone, it is also possible that they had a worse outcome because of receiving $\mathrm{AA}+\mathrm{P}$ later in the course of the disease [26]. In any case, the effect of adjustment was small and similar to that obtained using IPCW. Finally, because all patients eligible to switch did so, we could not apply the two-stage method, another common method for adjusting for switching. We also did not use iterative parameter estimation because it is a minor variation of RPSFTM and was not expected to provide significant additional information.

Although adjustment methods may provide estimates that are less biased than intent-to-treat analysis, they can be less precise, and the underlying assumptions may be difficult to verify $[18,19]$. However, the adjusted OS benefits were close to the OS benefits determined at the first interim analysis before treatment switching occurred. Overall, the adjustment had little impact because relatively few patients switched from placebo to $\mathrm{AA}+\mathrm{P}$ and because exposure to $\mathrm{AA}+\mathrm{P}$ was short.

In conclusion, this post hoc analysis confirmed that adding $\mathrm{AA}+\mathrm{P}$ to ADT in patients with newly diagnosed high-risk mCSPC reduces the risk of death by $37-38 \%$ and provides 17-18 months of additional survival. The combination of $\mathrm{AA}+\mathrm{P}$ and $\mathrm{ADT}$ adds to the options available to these patients who would otherwise survive for only around 3 years with ADT alone.

Acknowledgements Medical writing was provided by Phillip Leventhal, $\mathrm{PhD}$, of Evidera, and paid for by Janssen Pharmaceutica NV.

Author Contributions All authors helped interpret results, helped write or edit the manuscript, and approved the final version. N.J. Perualila, J. Diels, and T. Ito also helped conceive and design the study, and N.J. Perualila performed the analyses.

\section{Compliance with Ethical Standards}

Funding This study was funded by Janssen Pharmaceutica NV.

Conflicts of interest S Feyerabend has nothing to disclose. F. Saad reports grants, personal fees, and non-financial support from Janssen and Sanofi related to the conduct of the study, and grants, personal fees, and non-financial support from Astellas and Bayer unrelated to the submitted work. N.J. Perualila, S. Van Sanden, J. Diels, T. Ito, and P. De Porre are employees of Janssen. S. Van Sanden, T. Ito, and P. De Porre are shareholders of Janssen's parent company, Johnson \& Johnson. K. Fizazi reports financial support for participation in advisory boards for Amgen, AstraZeneca, Astellas, Bayer, Janssen, Sanofi, Orion, and CureVac.

Research involving human participants and/or animals All procedures performed in studies involving human participants were in accordance with the ethical standards of the local Institutional Review Boards for each site and with the 1964 Helsinki declaration and its later amendments or comparable ethical standards.

Informed consent Informed consent was obtained from all individual participants included in the LATITUDE study (ClinicalTrials.gov identifier NCT01715285).

Open Access This article is distributed under the terms of the Creative Commons Attribution-NonCommercial 4.0 International License (http://creativecommons.org/licenses/by-nc/4.0/), which permits any noncommercial use, distribution, and reproduction in any medium, provided you give appropriate credit to the original author(s) and the source, provide a link to the Creative Commons license, and indicate if changes were made.

\section{References}

1. Ferlay J, Soerjomataram I, Dikshit R, Eser S, Mathers C, Rebelo $\mathrm{M}$, et al. Cancer incidence and mortality worldwide: sources, methods and major patterns in GLOBOCAN 2012. Int J Cancer. 2015;136:E359-86.

2. Li J, Siegel DA, King JB. Stage-specific incidence rates and trends of prostate cancer by age, race, and ethnicity, United States, 20042014. Ann Epidemiol. 2018;28:328-30.

3. Jack RH, Davies EA, Moller H. Prostate cancer incidence, stage at diagnosis, treatment and survival in ethnic groups in South-East England. BJU Int. 2010;105:1226-30.

4. Oakley-Girvan I, Kolonel LN, Gallagher RP, Wu AH, Felberg A, Whittemore AS. Stage at diagnosis and survival in a multiethnic cohort of prostate cancer patients. Am J Public Health. 2003;93:1753-9.

5. Dong L, Zieren RC, Xue W, de Reijke TM, Pienta KJ. Metastatic prostate cancer remains incurable, why? Asian J Urol. 2019;6:26-41.

6. Purshouse K, Protheroe AS. Abiraterone acetate in combination with prednisone in the treatment of metastatic hormone-sensitive prostate cancer: clinical evidence and experience. Ther Adv Urol. 2019;11:1756287218820804.

7. Cassinello J, Arranz JA, Piulats JM, Sanchez A, Perez-Valderrama B, Mellado B, et al. SEOM clinical guidelines for the treatment of metastatic prostate cancer (2017). Clin Transl Oncol. 2018;20:57-68.

8. Fizazi K, Tran N, Fein L, Matsubara N, Rodriguez-Antolin A, Alekseev BY, et al. Abiraterone plus prednisone in metastatic, castration-sensitive prostate cancer. N Engl J Med. 2017;377:352-60.

9. James ND, de Bono JS, Spears MR, Clarke NW, Mason MD, Dearnaley DP, et al. Abiraterone for prostate cancer not previously treated with hormone therapy. N Engl J Med. 2017;377:338-51.

10. Feyerabend S, Saad F, Li T, Ito T, Diels J, Van Sanden S, et al. Survival benefit, disease progression and quality-of-life outcomes of abiraterone acetate plus prednisone versus docetaxel 
in metastatic hormone-sensitive prostate cancer: a network metaanalysis. Eur J Cancer. 2018;103:78-87.

11. Vale CL, Fisher DJ, White IR, Carpenter JR, Burdett S, Clarke NW, et al. What is the optimal systemic treatment of men with metastatic, hormone-naive prostate cancer? A STOPCAP systematic review and network meta-analysis. Ann Oncol. 2018;29:1249-57.

12. European Society for Medical Oncology. eUpdate-Cancer of the prostate treatment recommendations. 2019. https://www.esmo. org/Guidelines/Genitourinary-Cancers/Cancer-of-the-Prostate/ eUpdate-Treatment-Recommendation. Accessed 19 Nov 2019.

13. European Association of Urology. Prostate cancer. 2019. https:// uroweb.org/guideline/prostate-cancer/. Accessed 19 Nov 2019.

14. Janssen Pharmaceutical Company. ZYTIGA ${ }^{\circledR}$ Prescribing Information. 2019. http://www.janssenlabels.com/package-insert/produ ct-monograph/prescribing-information/ZYTIGA-pi.pdf. Accessed 19 Sep 2019.

15. Chi KN, Protheroe A, Rodriguez-Antolin A, Facchini G, Suttman $\mathrm{H}$, Matsubara N, et al. Patient-reported outcomes following abiraterone acetate plus prednisone added to androgen deprivation therapy in patients with newly diagnosed metastatic castrationnaive prostate cancer (LATITUDE): an international, randomised phase 3 trial. Lancet Oncol. 2018;19:194-206.

16. Fizazi K, Tran N, Fein L, Matsubara N, Rodriguez-Antolin A, Alekseev BY, et al. Abiraterone acetate plus prednisone in patients with newly diagnosed high-risk metastatic castration-sensitive prostate cancer (LATITUDE): final overall survival analysis of a randomised, double-blind, phase 3 trial. Lancet Oncol. 2019;20:686-700.

17. Latimer NR. Treatment switching in oncology trials and the acceptability of adjustment methods. Expert Rev Pharmacoecon Outcomes Res. 2015;15:561-4.

18. European Medicines Agency. Question and answer on adjustment for cross-over in estimating effects in oncology trials. EMA/845963/2018. London (UK): EMA; 2018.
19. Latimer NR, Abrams KR, Lambert PC, Crowther MJ, Wailoo AJ, Morden JP, et al. Adjusting survival time estimates to account for treatment switching in randomized controlled trials-an economic evaluation context: methods, limitations, and recommendations. Med Decis Making. 2014;34:387-402.

20. Robins JM, Tsiatis AA. Correcting for non-compliance in randomized trials using rank preserving structural failure time models. Commun Stat Theory Methods. 1991;20:2609-31.

21. Robins JM, Finkelstein DM. Correcting for noncompliance and dependent censoring in an AIDS Clinical Trial with inverse probability of censoring weighted (IPCW) log-rank tests. Biometrics. 2000;56:779-88.

22. Latimer NR, Bell H, Abrams KR, Amonkar MM, Casey M. Adjusting for treatment switching in the METRIC study shows further improved overall survival with trametinib compared with chemotherapy. Cancer Med. 2016;5:806-15.

23. Latimer NR, Amonkar MM, Stapelkamp C, Sun P. Adjusting for confounding effects of treatment switching in a randomized phase II study of dabrafenib plus trametinib in BRAF V600 + metastatic melanoma. Melanoma Res. 2015;25:528-36.

24. Latimer NR, Abrams KR, Amonkar MM, Stapelkamp C, Swann RS. Adjusting for the confounding effects of treatment switching - the BREAK-3 trial: dabrafenib versus dacarbazine. Oncologist. 2015;20:798-805.

25. Skaltsa K, Ivanescu C, Naidoo S, Phung, Holmstrom S, Latimer NR. Adjusting overall survival estimates after treatment switching: a case study in metastatic castration-resistant prostate cancer. Target Oncol. 2017;12:111-21.

26. Saad F. Importance of early treatment in metastatic prostate cancer: a question of life or death. Lancet Oncol. 2019;20:609-11. 